\title{
Effects of task duration on sensitivity to sleep deprivation using the multi-attribute task battery
}

\author{
JOHN A. CALDWELL and STEPHANIE RAMSPOTT \\ U.S. Army Aeromedical Research Laboratory, Fort Rucker, Alabama
}

\begin{abstract}
Several factors affect the sensitivity to sleep deprivation in the performance of computerized tests. Two of these are task complexity and duration. Complexity can improve sensitivity by increasing the demands required to complete the task, but it can also decrease sensitivity by improving the subject's motivation. The effects of task duration are more predictable in that longer tests generally are more susceptible to deprivation effects than shorter ones. The impact of task duration on an interesting, but complex, aviation simulation was examined here. By breaking down data from 30-min multi-attribute task battery administrations into the first, second, and third $10 \mathrm{~min}$ of performance, it was shown that tests shorter than 30 min underestimate the impact of sleep loss on performance. This was especially evident in measures of time-out and tracking errors.
\end{abstract}

It is well accepted that sleep deprivation (SD) produces a wide array of performance decrements, including increased reaction times, decreased vigilance and alertness, distortions in cognition and perception, and degraded mood (Krueger, 1989). However, a review of individual studies in the literature shows substantial variation in the effects of sleep loss from one investigation to another. It is likely that discrepancies among studies are due to a number of design and/or methodological factors rather than to differences in the effects of sleep loss per se.

One factor that contributes to disparate findings across SD studies (which is of particular importance in partial deprivation or napping studies) is the number of minutes between the time of awakening and the time of testing. Wilkinson and Stretton (1971), for instance, found that reaction time, arithmetic calculations, and psychomotor coordination were particularly impaired when subjects were tested within $15 \mathrm{~min}$ of being awakened from sleep. This is a phenomenon referred to as sleep inertia, or the tendency toward postsleep impairment and disorientation that is especially severe after a brief period of sleep inserted into a prolonged period of wakefulness (Dinges, 1989). If testing is always performed immediately upon awakening from sleep, it could erroneously be concluded that, from a performance standpoint, even a full night's sleep is worse than total SD. Recent investigations in our laboratory attest to this problem by showing that strategic napping (compared to total SD) substantially impaired mood states immediately upon awakening, despite the fact that napping markedly improved mood, alertness, and per-

The authors thank Susan J. Gardner for her expert assistance in the analysis of the data and the preparation of this report. Correspondence concerning this article should be addressed to J.A. Caldwell, U.S. Army Aeromedical Research Laboratory, P.O. Box 620577, Fort Rucker, AL 36362-0577 (e-mail: caldwell(@)rucker-emh2.army.mil) formance later in the deprivation period (Caldwell et al., 1997).

A second factor is the amount of feedback given to sleep-deprived subjects. Generally, feedback seems to improve performance by augmenting motivation levels. Wilkinson (1961) found that knowledge of results is especially important for maintaining the performance of sleep-deprived subjects. Feedback significantly attenuated the effects of one night of total SD on performance of a five-choice test of serial reaction.

A third factor is the type of task chosen for the research approach. It has been found that some tasks are more likely than others to be affected by SD. Wilkinson (1964), for instance, reported that, whereas serial reaction time and vigilance tests were significantly degraded by sleep loss, rote learning and performance on various games were not. He attributed these differences to such factors as task complexity and the subjects' interest in the tests. Generally, the more complex and less interesting the task, the more performance suffered after sleep loss. However, if the task was both complex and interesting, the subjects' interest levels apparently provided enough intrinsic incentive to overcome the degrading effects of complexity-a finding that has received support from Elsmore et al. (1995) and others.

A final factor that accounts for discrepancies across SD studies is test duration. It has been found that performance on most tasks can be adequately maintained for brief periods of time (i.e., 5-10 min), even in subjects who are significantly sleep deprived; but when task durations are extended to $20-30 \mathrm{~min}$, performance deteriorates rapidly (Wilkinson, 1969b; Wilkinson, Edwards, \& Haines, 1966). Recently completed research in which the synthetic work battery (SYNWORK) was administered to sleep-deprived aviators in a sustained work scenario (Caldwell et al., 1995; Caldwell et al., 1994; Caldwell 


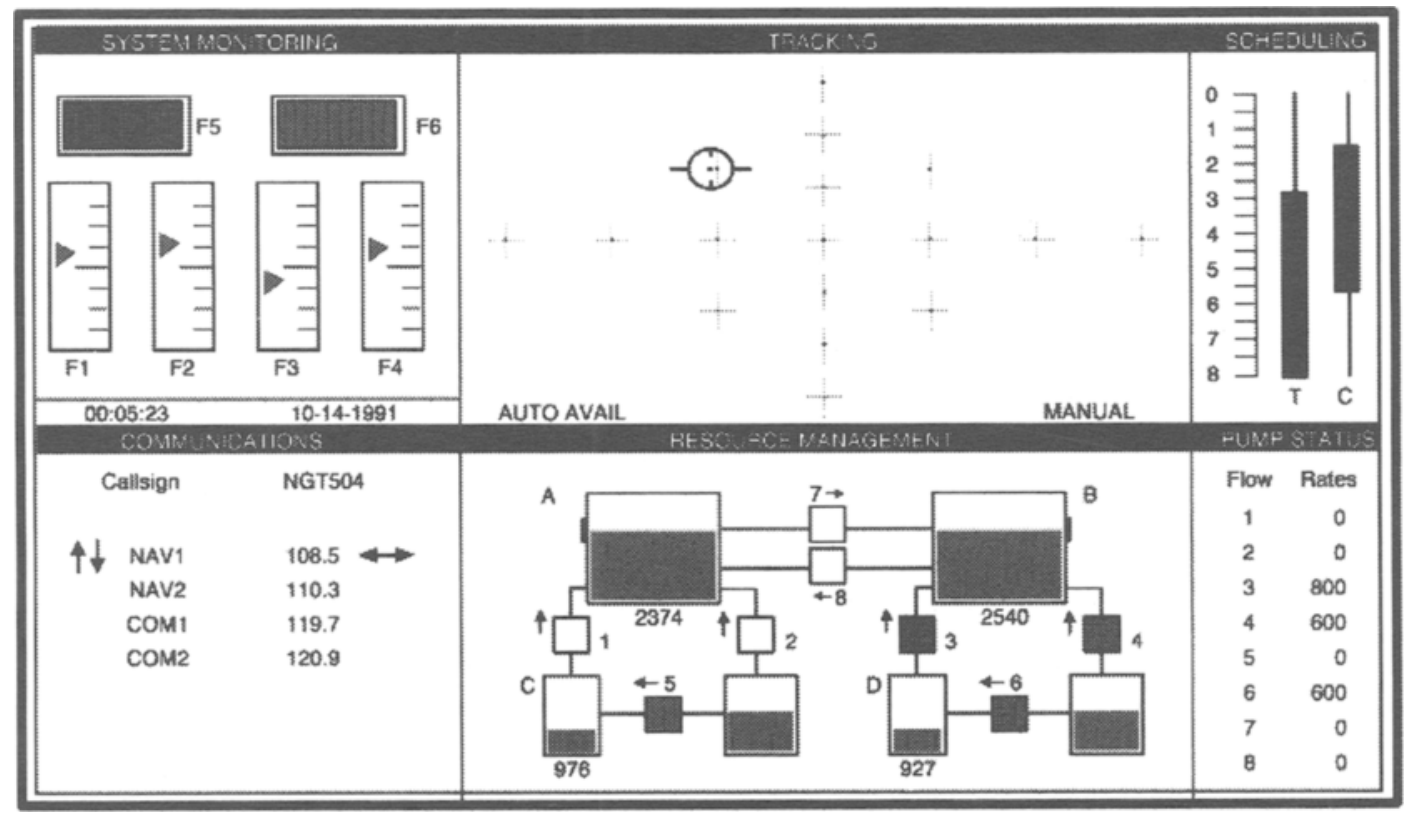

Figure 1. The layout of the multi-attribute task battery, as it was presented on the computer screen.

et al., 1997) tends to support this contention. In the first two studies, the effects of placebo versus dextroamphetamine on the performance of sleep-deprived subjects were examined, using 10-min administrations of SYNWORK placed at 4-h intervals. Although this task is one that requires substantial sharing of cognitive resources (among simultaneously presented memory, arithmetic, visual monitoring, and auditory monitoring components), no drugrelated performance differences were found in either of the two studies, with the exception of a single drug $\times$ session interaction on 1 variable out of 11 (performance on only one subtask out of the four was affected). In a later study of the effects of strategic naps versus total SD, the duration of SYNWORK was increased to $20 \mathrm{~min}$, and the same basic testing schedule was repeated. This time, results indicated intervention-related effects on three of the four subtasks, despite the fact that only basic composite scores (instead of the more sensitive individual measures used in the earlier studies) were analyzed. Thus, lengthening the test duration from 10 to $20 \mathrm{~min}$ appeared to substantially increase task sensitivity.

In summary, differences in elapsed times from awakening, knowledge of results, task sensitivity, and task duration, as well as other factors, may account for inconsistencies in the published literature regarding the impact of SD on both mood and performance. It is unlikely that sleep loss is such a minor stressor that it produces decrements only in specific subgroups or in certain circumstances. Instead, it is more plausible that SD exerts a universally negative effect on humans that is difficult to quantify reliably because of differences across studies. In fact, a meta-analysis by Pilcher and Huffcutt (1996) suggests that methodological inconsistencies result in a general underestimation of the impact of SD in humans. The precise effects of test-specific factors remain unclear, because complex interactions no doubt exist. For instance, although it is fairly certain that increasing task duration will improve task sensitivity to SD, it is less obvious whether this will apply equally to both complex and simple tasks.

The present study was conducted to more clearly delineate the effects of task duration on sensitivity to SD in circumstances in which a complex, multitask test battery (specifically, the multi-attribute task battery, MATB) is used. To address the time-on-task issue, 30-min blocks of test administrations were used. These blocks were subsequently broken into three successive 10 -min intervals for purposes of analysis. The factor of sleep inertia (time since awakening) was controlled by testing subjects continuously, with no sleep during the latter part of a 38-h period of sustained wakefulness (subjects all had been awake for at least $13 \mathrm{~h}$ prior to the first test). To minimize confounds regarding knowledge of results, feedback was not provided to any of the subjects (a future study will establish the impact of this factor in a similar context). The MATB was selected on the basis of the fact that (1) it requires a high level of cognitive resource sharing, and (2) it has been rated by subjects tested in this laboratory over the past several years as a good, face-valid method for assessing aviator performance. Both rated aviators and 


\section{Communications Monitoring Task}
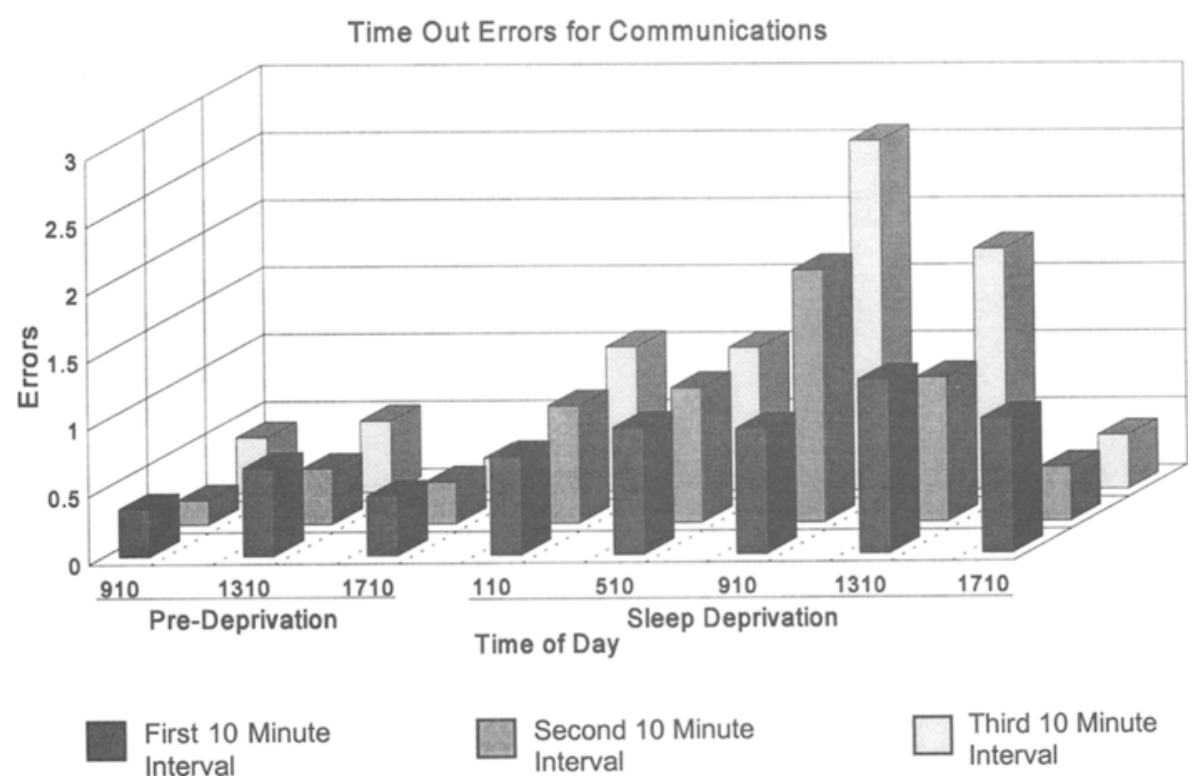

Figure 2. Effects of interval and time on time-out errors in communications. Note: The darkest bars show performance in the first 10-min interval, and the lightest bars depict performance in the third 10-min interval (the same scheme applies to all of the interaction figures).

flight students find the MATB challenging and interesting, since it is a complex aviation simulation.

\section{METHOD}

\section{Subjects}

Eighteen males (rated aviators and flight students) between the ages of 22 and 31 (mean age of 24.4) participated after prescreening and medical evaluation. One subject's data were excluded because of computer problems. Candidate subjects who used tobacco, currently took medications (other than ibuprophen, aspirin, acetominophen, or sodium naproxin), consumed more than three 8ounce cups of coffee or five 12-ounce caffeinated soft drinks per day, or experienced a current medical disorder (including sleep abnormalities) were excluded. Females were not tested because none volunteered. The subjects refrained from alcohol and other drugs during the protocol.

\section{Apparatus}

The MATB is a computer-based, aviation-related, synthetic task battery that was initially developed by NASA researchers (Comstock \& Arnegard, 1992). The test was implemented on a 486 desktop computer equipped with a game card (Gamecard 3, C.H. Products), a voice synthesizer card (Soundblaster 16, Creative Lab.). stereo headphones (Sony), a joystick (Advance Gravis Computer Tech. LTD), and a standard keyboard and color monitor

\section{Procedure}

The MATB included a resource (fuel) management task, a communications task, a systems-monitoring task, and an unstable tracking task, each of which was presented in a separate quadrant of the computer screen (see Figure 1). The subjects were instructed that they would be completing tasks designed to assess their performance in a simulated flight environment. Specifically, they were told that they would be performing a tracking task while simultaneously monitoring system status and communication channels and managing fuel resources. The subjects were not provided with any instructions about the relative importance of any one task over another task, nor were they advised about how they should attempt to divide their attention among the different subtasks. Instructions for the individual subtasks were as follows:

The system monitoring task will require attending to the 4 gauges marked $F 1, F 2, F 3$, and $F 4$; and the two boxes marked $F 5$ (usually green) and F6 (usually blank) on the computer screen. Use the corresponding keys to manipulate the boxes and gauges. Press the $F 5$ key immediately if the F5 box is no longer green. The F6 box should always be blank. Press the $F 6$ key if the F6 box turns red. The pointers in the gauges need to be within one tick mark above or below the mid-line. Press the corresponding keys immediately if the pointers move beyond the one-tick-mark range. When this out-of-range status is correctly identified, the pointer will move immediately back to the mid-line and a bar at the bottom of the gauge will be illuminated in yellow.

The tracking task should be executed using the joystick to keep a target in the center of its window within the dotted lines that form a rectangle. This is an attempt to simulate the demands of manual control.

The communications task simulates receiving audio messages from Air Traffic Control through a set of headphones. You will respond only to the call sign "NGT504" and make appropriate frequency changes on a Navigation and/or Communication radio. Your call sign will be displayed at the top of the Communications window and you will need to discriminate your call sign from other three-letter, threenumber combinations. A command to change frequency will only be repeated once. Use $U p$ and Down arrow keys on the keyboard to move from "NAV1" through "COM2." Use Left and Right arrow keys on the keyboard to change frequency. The Left arrow key will decrease the frequency; the Right arrow key will increase the frequency. Every keystroke will result in a $0.2-\mathrm{MHz}$ change in radio frequency. Press the Enter key to acknowledge the completed frequency adjustments

The goal of the resource (fuel) management task is to maintain tanks $A$ and $B$ at 2,500 units each, which is indicated by numbers below the ranks. This desired level is also indicated by a tick mark in the 


\section{Communications Task}

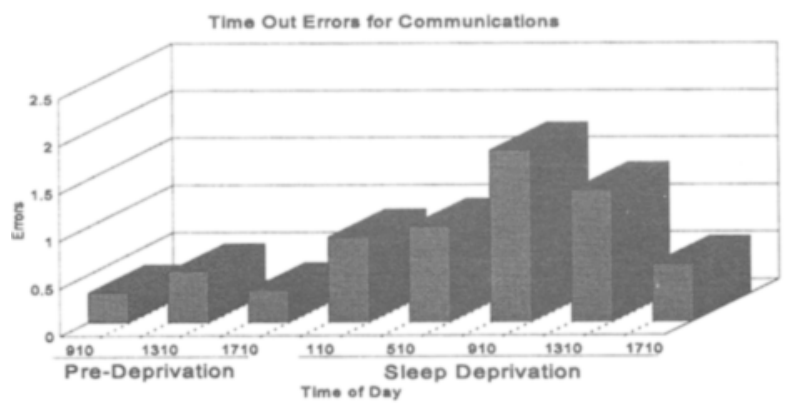

Systems Monitoring Task

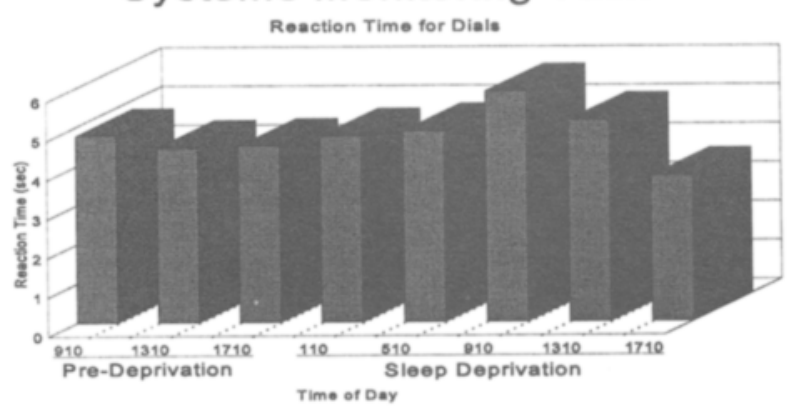

Systems Monitoring Task

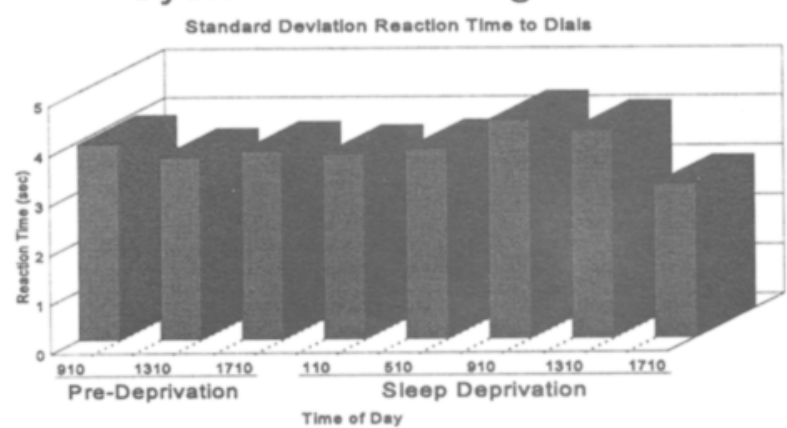

Systems Monitoring Task

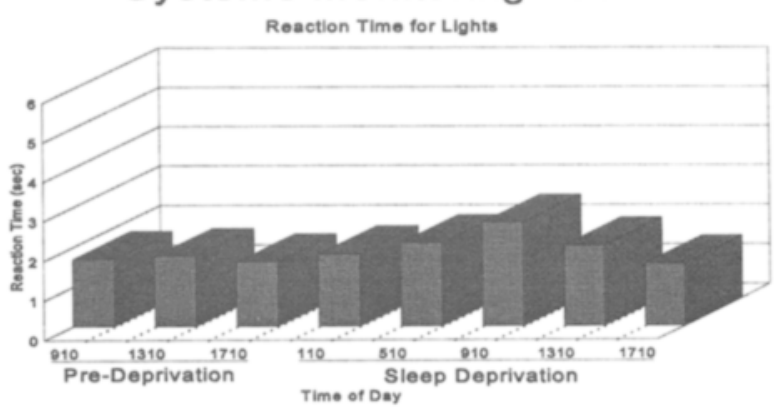

Systems Monitoring Task

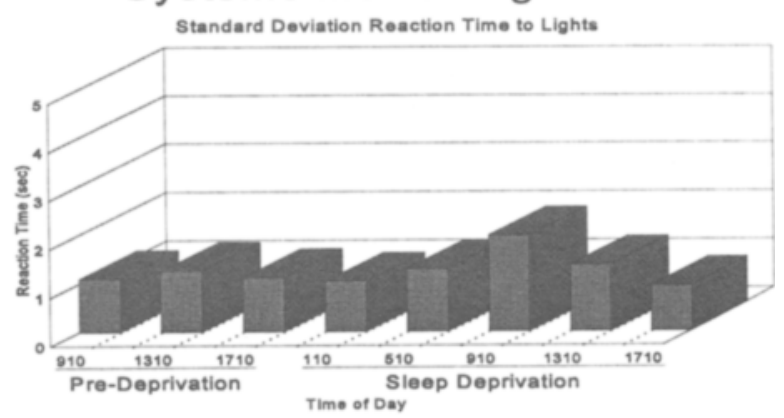

Systems Monitoring Task

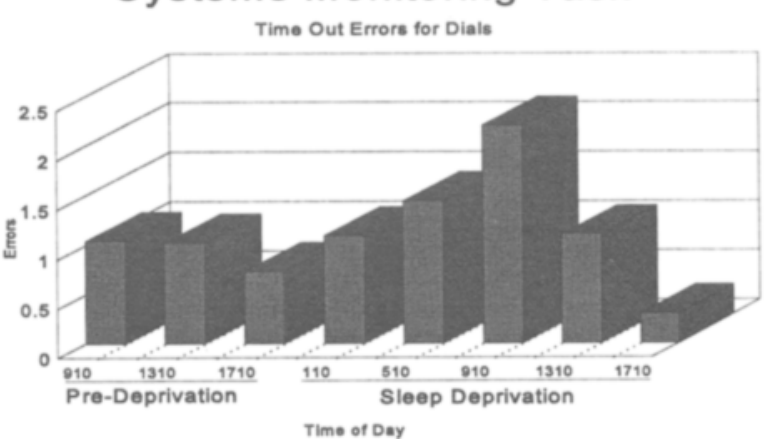

Figure 3. Effects of testing time (with intervals collapsed) on time-out errors in communications and both reaction time measures and time-out errors in systems monitoring.

shaded bar on the sides of the two tanks. The shaded region surrounding the tick mark represents acceptable performance. The resource management task can be accomplished by turning on or off any of the pumps labeled 1 through 8 . Periodically, a pump failure will occur and the pump will turn red; this pump cannot be turned back on until the red light goes out. The process of transferring fuel is accomplished by activating the pumps using corresponding number keys. Pressing the number key a second time will turn that particular pump off. When the pump is actively transferring fuel, it will turn green, and the direction of transfer is indicated by arrow keys. The maximum capacity for tanks $A$ and $B$ is 4,000 units, and for tanks $C$ and D, the maximum capacity is 2,000 units. The remaining two supply tanks have an unlimited capacity. The flow rates for each pump are shown in the "Pump Status" window. However, you do not manipulate the pump status; it is simply used as a gauge.

In the resource (fuel) management task, either Pump 2 or Pump 4 failed once every $2 \mathrm{~min}$. In the systems-monitoring task, there was either a dial or light indication requiring a response from the subject three times per minute. In the communications task, radio messages were delivered at a rate of two messages per minute. A response was required for half of these messages.

\section{Testing Schedule}

All test subjects resided in the laboratory throughout the experiment. After initial training, there was a full 10 -h night of sleep prior to actual testing. Upon awakening (at 0800 ), the subjects completed predeprivation (PD) testing at 4-h intervals (at 0910, 1310, and 1710) prior to an evening in which they engaged in light physical activity, watched television, and interacted with staff members but were not allowed to sleep. At 0110 , the subjects began deprivation testing, and other sessions followed at 0510,0910,1310, and 1710 . Each test administration was $30 \mathrm{~min}$ long. A variety of test activities (mood and alertness tests) occurred between each of the MATB 


\section{Systems Monitoring Task}

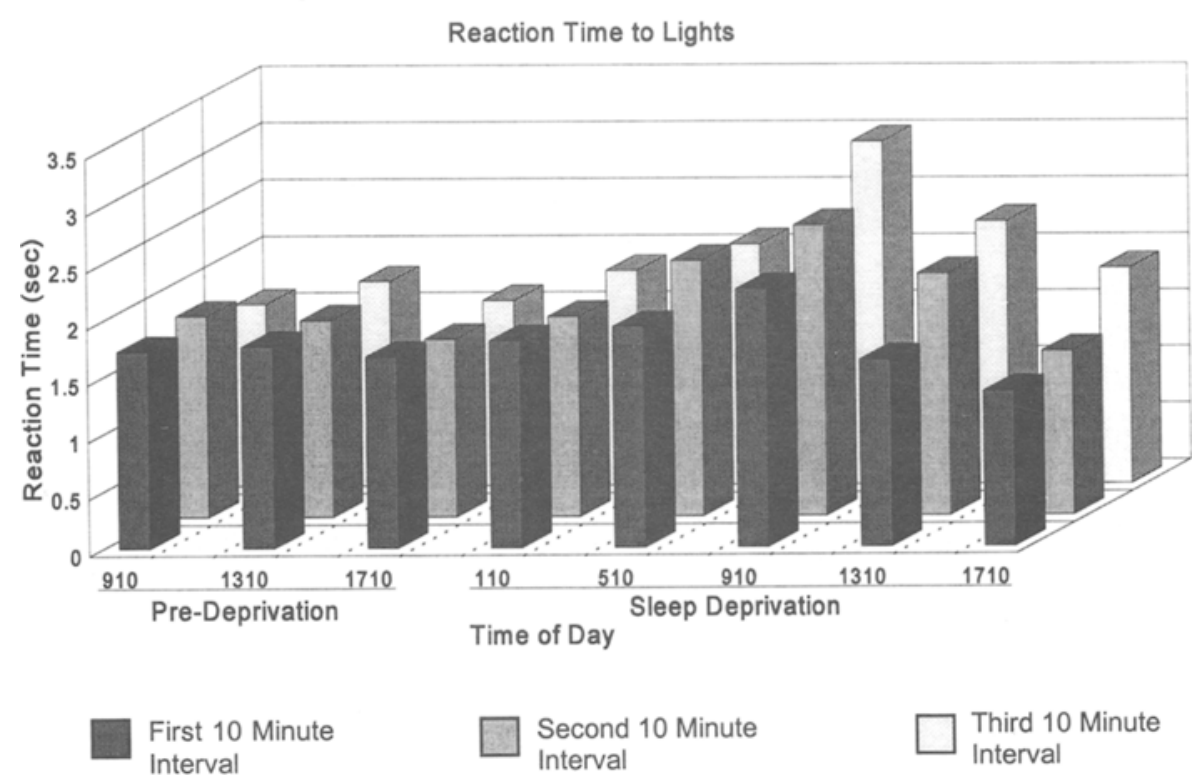

Figure 4. Effects of interval and time on the reaction time for lights in systems monitoring.

administrations, and a complete schedule has been published elsewhere (Caldwell et al., 1997).

\section{Data Analysis}

A computer program was used to extract a number of performance indices from each MATB administration. The deviation of fuel levels from the ideal value of 2,500 units in the fuel management task; the reaction time, standard deviation of reaction time, and time-out errors in the communications task; the reaction times to lights and dials, the standard deviations of reaction times to lights and dials, and time-out errors for lights and dials in the systemsmonitoring task; and the root mean square (RMS) tracking errors in the unstable tracking task were saved for analysis.

The data from each iteration of the test were segmented into three intervals (first $10 \mathrm{~min}$, second $10 \mathrm{~min}$, and third $10 \mathrm{~min}$ ) for each of the four tasks (fuel management, systems monitoring, etc.). Twoway analyses of variance were used to examine differences attributable to interval (first, second, and third $10 \mathrm{~min}$ ) and time (PD0910, 1310, and 1710; deprivation-0110,0510, 0910,1310, and 1710). Both PD and deprivation data were included in each analysis in order to ensure that any observed changes in task sensitivity were a function primarily of SD rather than simply of time of day (Chmiel, Totterdell, \& Folkard, 1995).

\section{RESULTS}

\section{Fuel Management}

There were no significant main effects or interactions on the mean deviation of fuel units from 2,500 (the ideal) in the fuel management task. Apparently, the withinsubjects variability in responding was so great that it overshadowed any deprivation effects.

\section{Communications}

There was an interaction between interval and time (test session) on time-out errors in the communications task $[F(14,224)=2.60, p=.0016]$, but neither the reaction time for correct responses nor the standard deviation of reaction times was similarly affected. The interval $x$ time interaction was due to variations in the pattern of differences among the various testing times within the first, second, and third 10 -min intervals $(p<.05)$. Pairwise contrasts indicated that none of the PD conditions differed from one another at any of the intervals, but instead, the differences all occurred between PD and SD sessions or within the deprivation sessions themselves (see Figure 2). In the first $10 \mathrm{~min}$, time-out errors were fewer at PD 0910 and PD 1710 than at SD 1310 or SD 1710 and fewer at PD 0910 than at SD 0510 . In the second $10 \mathrm{~min}$, time-out errors were fewer at PD 0910, PD 1310, and PD 1710 than at SD 0910; errors were fewer at PD 0910 than at SD 1310; and errors were larger at both the SD 0910 and the SD 1310 times than at SD 1710 . In the third $10 \mathrm{~min}$, time-out errors were lower at all three PD sessions than at SD 0910 and SD 1310; and, in addition, they were lower at PD 1710 than at SD 0110 and SD 0510, whereas they were higher at both SD 0910 and SD 1310 than at SD 1710. Generally, SD effects became more apparent as a function of test duration. A main effect of time on time-out errors $[F(7,112)=$ $4.08, p=.0005$ ] showed that errors (with intervals collapsed) were less numerous at both PD 0910 and PD 1310 than at SD 0910; errors were less numerous at PD 1310 


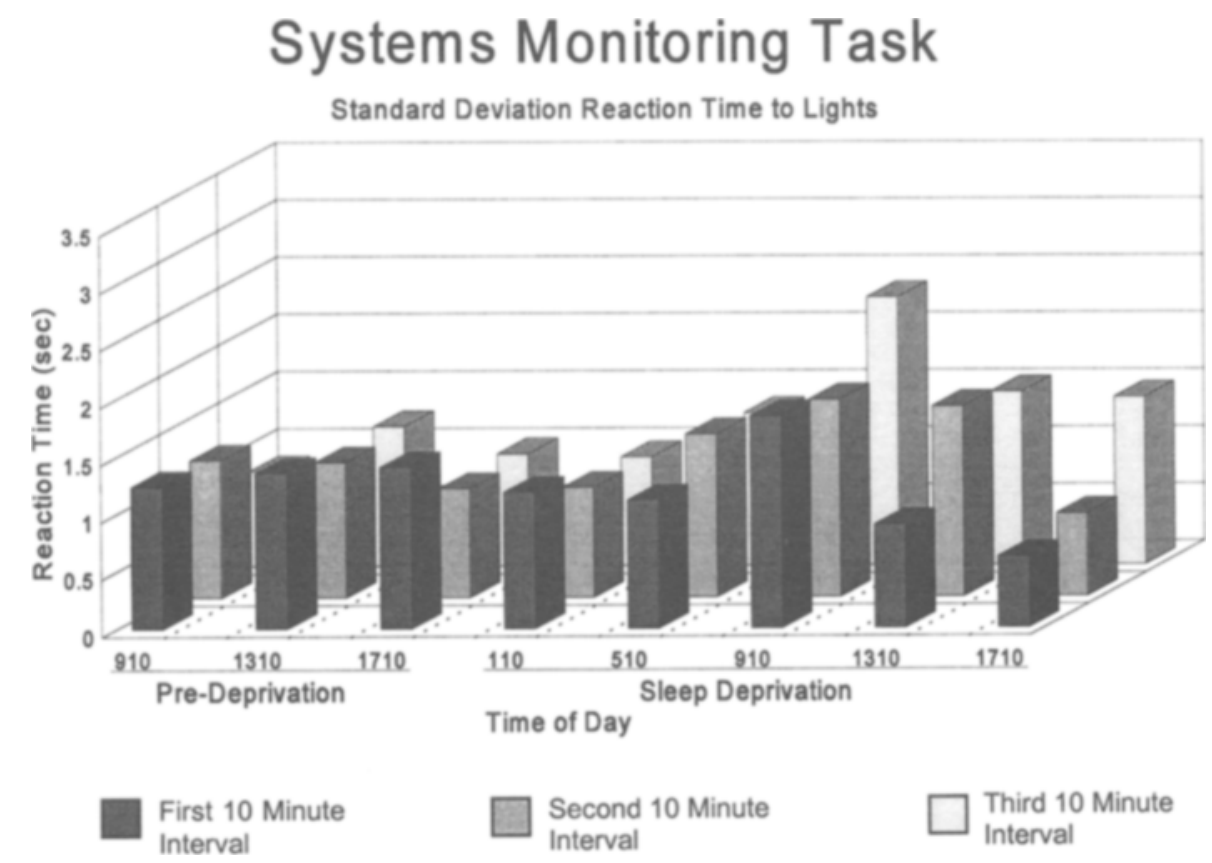

Figure 5. Effects of interval and time on the standard deviation of reaction times for lights in systems monitoring.

than at SD 1310; and errors were less numerous at PD 1710 than at any of the SD sessions, with the exception of SD $1710(p<.05)$. This main effect, along with the main effects of time from other subtests, is shown in Figure 3. There were no main effects on the interval factor.

\section{Systems Monitoring}

There were interval $\times$ time interactions on the reaction time for lights $[F(14,224)=2.10, p=.0126]$, the standard deviation of reaction times for lights $[F(14,224)=2.57$, $p=.0019]$, time-out errors for lights $[F(14,224)=1.80$, $p=.0391]$, and time-out errors for dials $[F(14,224)=$ $2.64, p=.0014]$. Analysis of simple effects indicated that there were differences within each interval (first, second, and third $10 \mathrm{~min}$ ) across the PD and deprivation sessions for both of the reaction time measures $(p<.05)$. Generally, deprivation-related changes became more noticeable as the duration of the task increased. There were no differences among the test sessions that preceded SD. Within the first 10-min interval, although none of the PD sessions differed from one another, reaction times were longer at SD 0910 than at PD 0910 or PD 1710, whereas they were shorter at SD 1710 than at PD 0910 or PD 1310. Within the SD sessions, reaction times were slower at SD 0510 and SD 0910 than at SD 1310 or SD 1710. Also, they were slower at SD 0110 than at SD $1710(p<.05)$. Within the second 10-min interval, reaction times were slower at SD 0910 than at any of the PD sessions or any of the SD sessions, with the exception of SD 0510. Reaction times at SD 0510 were longer than those at PD 1710 or SD 1710; and reaction times at SD 1310 were longer than those at $\mathrm{PD}$ 1710 , SD 0110 , or SD $1710(p<.05)$. Within the third 10-min interval, reaction times were slower at SD 0910 than at any of the PD sessions or any of the SD sessions, with the exception of SD 1310; slower at SD 0510 and SD 1310 than at PD 0910 or PD 1710; and slower at SD 1310 than at $\mathrm{SD} 1710(p<.05)$. These reaction time effects are shown in Figure 4. The pattern of the standard deviation data was somewhat similar, but the overall number of effects was smaller. Within the first 10-min interval, almost all of the other sessions (with the exception of SD 0510 and SD 1310) were found to be more variable than the SD 1710 session, whereas all of the SD sessions were less variable than the SD 0910 session $(p<.05)$. Within the second 10-min interval, the SD 0510, SD 0910, and SD 1310 sessions were more variable than the SD 1710 session, and all of the PD sessions, as well as the SD 0110 and SD 1710 sessions, were less variable than the SD 0910 session. SD 1310 was more variable than PD 1710 or SD 0110 . Within the third 10-min interval, the variability in SD 1710 had increased so that only SD 0910 was greater, but the variability at SD 0910 was higher than the variability at any of the PD or SD sessions. Also, the variability in reaction times at SD 0510 was greater than the variability at PD 0910 , PD 1710 , or SD $0110(p<$ .05 ). These effects on the standard deviation of reaction times are depicted in Figure 5.

The interval $\times$ time interaction for time-out errors for dials was attributable to the fact that there were differences among the testing times only within the first and 


\section{Systems Monitoring Task}

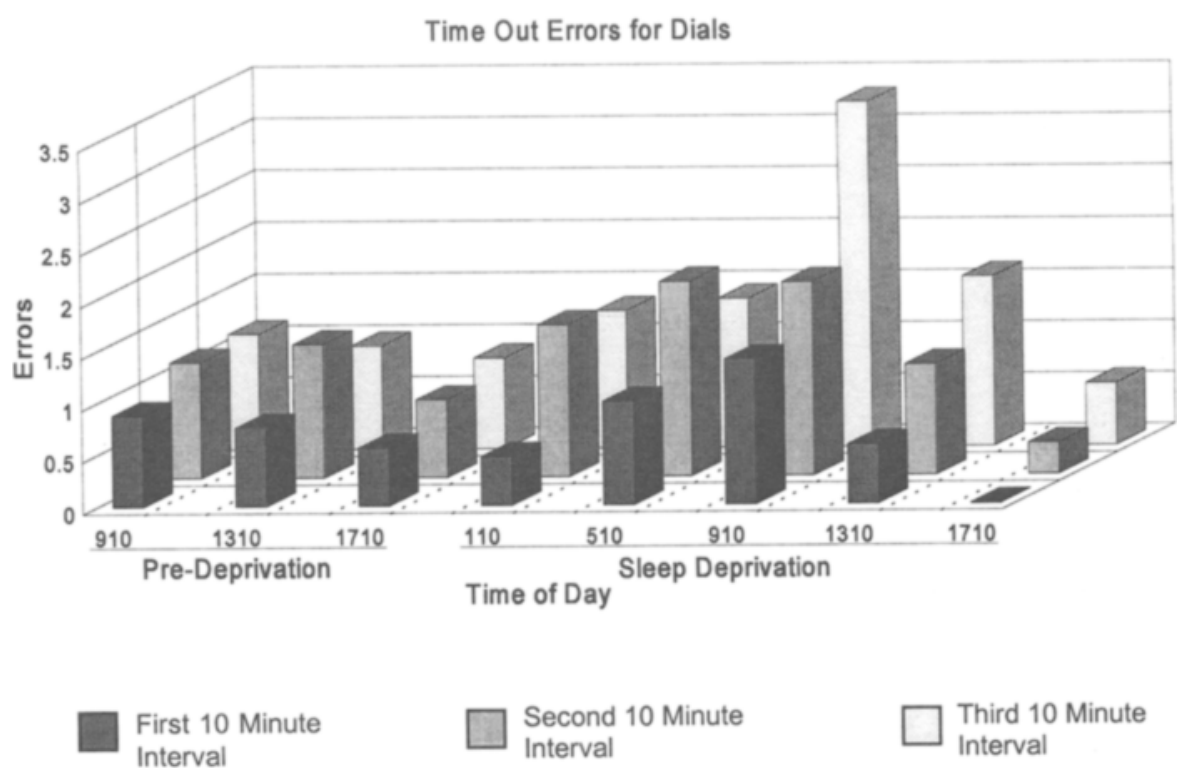

Figure 6. Effects of interval and time on time-out errors for dials in systems monitoring.

the third 10 -min intervals $(p<.05)$ but not within the second. As was the case with the reaction time measures, the general trend was toward larger discrepancies between PD and deprivation sessions as task duration increased from 10 to $30 \mathrm{~min}$. There were no differences among the PD performance scores. Within the first 10 min interval, the only effects were due to smaller timeout errors during the SD 1710 session than during all of the other sessions $(p<.05)$, with the exception of PD 1710 and SD 1310 (where no differences occurred). Within the second 10-min interval, there were no significant effects, as has already been noted. Within the third 10-min interval, the general trend toward poorer performance at 0910 during SD (SD 0910) became evident, as errors at this time were significantly more frequent than errors at any of the PD sessions or any of the SD sessions, with the exception of SD $1310(p<.05)$. The effects on time-out errors for dials are shown in Figure 6. The interaction on time-out errors for lights was attributable to the presence of a marginally significant difference across the testing times only in the third 10 -min interval ( $p=.0551)$ but not in the first or second. As can be seen in Figure 7, this effect was primarily due to inordinately poor performance at SD 0910 (most clearly evident only after 30 min of task duration).

In addition to these interactions, there were main effects of time on the reaction times to lights $[F(7,112)=$ $7.36, p<.0001]$ and dials $[F(7,112)=6.32, p<.0001]$, the standard deviations for correct responses to lights
$[F(7,112)=5.76, p<.0001]$ and dials $[F(7,112)=3.53$, $p=.0018]$, and the time-out errors to dials $[F(7,112)=$ $2.80, p=.0100]$. The reaction times to both lights and dials were slower at SD 0910 than at any of the PD or deprivation sessions. In addition, reaction time to lights was slower at SD 0510 than at PD 1710 and slower at SD 1310 than at PD 0910, PD 1710, or SD 1710. Reaction time to dials was slower at SD 0510 than at SD 0110 or SD 1710 and faster at SD 1710 than at PD 0910, SD 0110 , or SD 1310. The reaction time to dials also was slower at the 1310 deprivation session than at the PD 1310 session $(p<.05)$. The standard deviation for correct responses to lights was larger at SD 0910 than at any other session (PD or deprivation), larger at SD 0510 than at SD 0110, and larger at SD 1310 than at SD $1710(p<.05)$. The main effect on standard deviation for correct responses to dials was due to the small variability at SD 1710 in comparison with all of the other sessions (PD and deprivation) and a slight reduction in variability from the first to the second PD sessions $(p<.05)$. The main effect on time-out errors for dials was due to more frequent errors at SD 0910 than at PD 1710 or SD 1710, whereas the errors at SD 1710 were lower than those at PD 0910 and SD $1310(p<.05)$.

There were main effects on the interval factor for the reaction times to lights $[F(2,32)=6.25, p=.0051]$ and dials $[F(2,32)=8.94, p=.0008]$ and the time-out errors for lights $[F(2,32)=4.63, p=.0171]$ and dials $[F(2,32)=$ $10.86, p<.0003]$. In every case, performance was worse 


\section{Systems Monitoring Task}

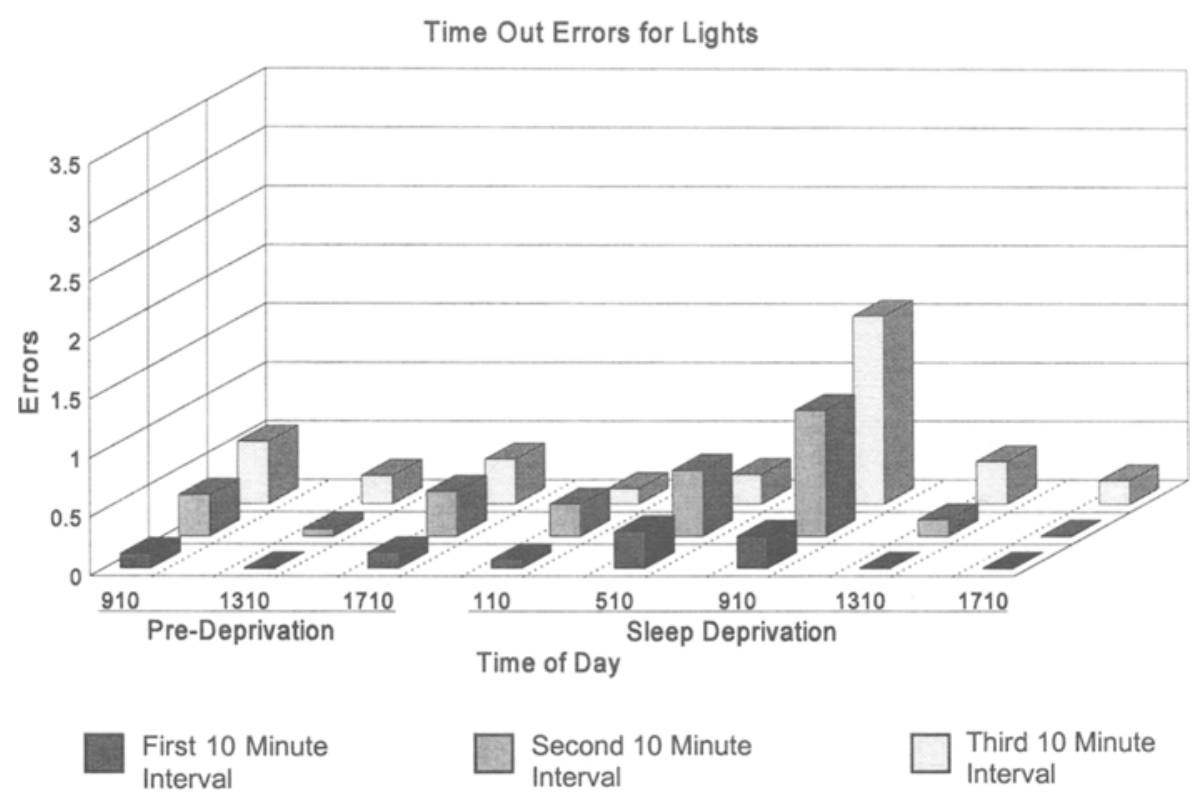

Figure 7. Effects of interval and time on time-out errors for lights in systems monitoring.

(i.e., longer reaction times and increased errors) in the third 10-min interval than in the first; in every case, except for the reaction time for lights, performance was worse in the second 10-min interval than in the first; and for reaction time to dials, performance was worse in the third interval than in the second $(p<.05)$. These interval effects are shown in Table 1.

\section{Tracking}

There was an interval $\times$ time interaction $[F(14,224)=$ $3.62, p<.0001]$, a main effect of time $[F(7,112)=6.71$, $p<.0001]$, and a main effect of interval $[F(2,32)=19.74$, $p<.0001]$ on tracking RMS errors. Although there were differences among the test sessions at every interval length, the interaction was attributable to a deprivationrelated worsening in the pattern of performance as a function of time on task $(p<.05)$. There were no differences in tracking performance within the PD period. In the first 10-min interval, performance in the SD 0910 session was worse than in all of the other sessions, with the exception of SD 0510 , and the SD 0510 session was

Table 1

Effects of Task Duration (First, Second, and Third 10-Min Interval) on Performance

\begin{tabular}{lccc}
\hline \multicolumn{1}{c}{ Variate } & First $10 \mathrm{~min}$ & Second $10 \mathrm{~min}$ & Third $10 \mathrm{~min}$ \\
\hline Reaction time, lights & 1.78 & 1.90 & 2.04 \\
Reaction time, dials & 4.47 & 4.76 & 5.08 \\
Time-out, lights & 0.11 & 0.35 & 0.46 \\
Time-out, dials & 0.71 & 1.22 & 1.42 \\
\hline
\end{tabular}

worse than PD 1310 and SD 0110 as well. Performance at SD 1310 was poorer than performance at PD 1310 or SD $1710(p<.05)$. In the second 10 -min interval, performance at SD 0910 was worse than at all of the other sessions, without exception. Although performance at SD 0510 was more impaired, as compared with the first 10-min interval, tracking skill at SD 1310 was much lower than at any of the other sessions, with the exception of SD 0510 and SD $0910(p<.05)$. In the third 10-min interval, tracking at SD 0910 was poorer than tracking at every session, with the exception of SD 1310 (at which time tracking had deteriorated almost as much as it had at SD 0910), and tracking at SD 0510 was worse than tracking at PD 1310 and SD 0110 . In addition, performance was lower at SD 1310 than at PD 0910, PD 1310, PD 1710, SD 0110 , and SD $1710(p<.05)$. These effects are shown in Figure 8.

The main effect on the time factor occurred because tracking was worse at SD 0910 than at any other time and worse at SD 1310 than at all three PD sessions, SD 0110 , and SD 1710. In addition, performance at SD 0510 was worse than at PD 1310 or SD $0110(p<.05)$. The main effect on the interval factor was due to a progressive worsening of performance from the first to the third $10-\mathrm{min}$ interval (all comparisons were statistically significant).

\section{DISCUSSION}

This investigation showed that performance on a 30 min aviation simulation task during $38 \mathrm{~h}$ of continuous 


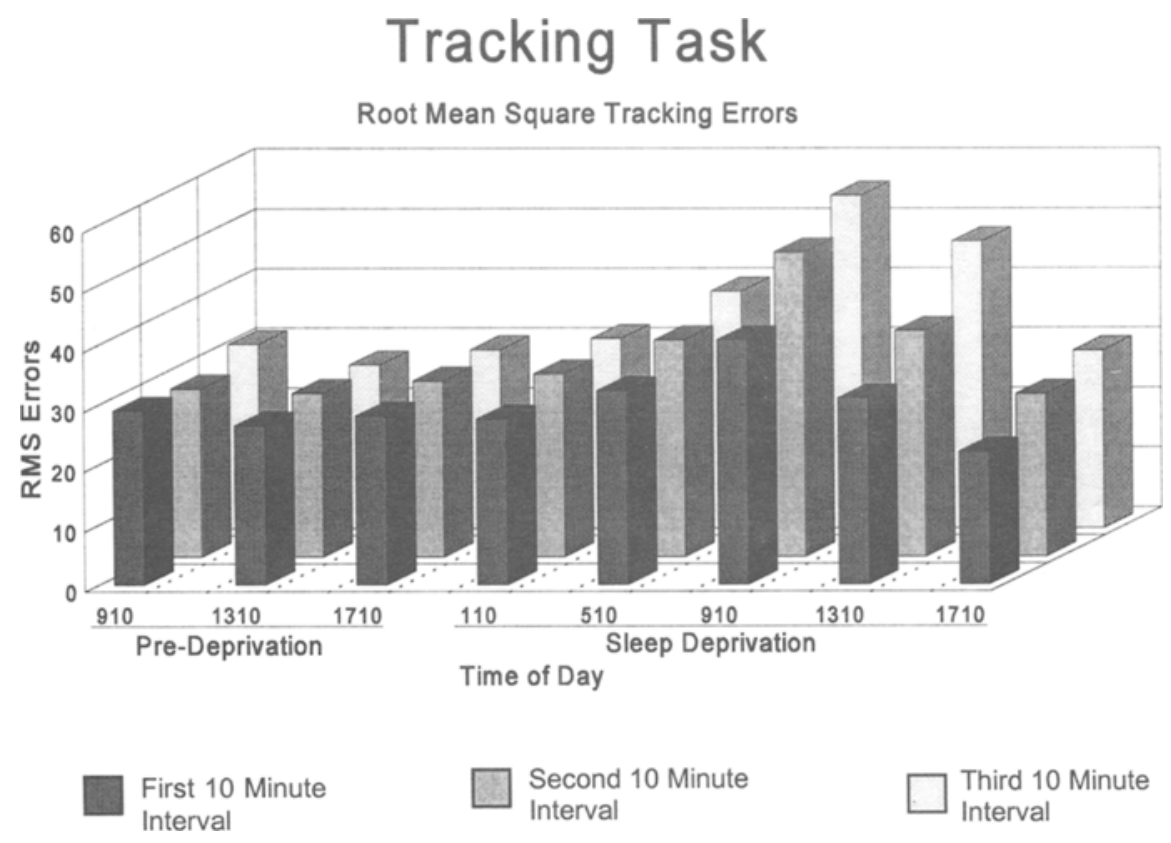

Figure 8. Effects of interval and time on root mean square errors in tracking.

wakefulness declined most severely in the midmorning after one night of sleep loss. Furthermore, the study revealed that, if a shorter task duration had been selected, the overall impact of SD on performance would have been seriously underestimated (or missed altogether), despite the fact that a rather complex sharing of mental resources was required by the selected task (the MATB). An examination of 11 performance variates (i.e., reaction-time measures, errors, etc.) indicated that task durations of 10 , 20 , and 30 min clearly were differentially sensitive to the effects of sleep loss. Particularly noticeable were the increases in reaction times (responding to communications calls and warning lights), time-out errors (responding to warning lights and dial deviations), and tracking errors, all of which became more pronounced as task duration increased from 10 to $30 \mathrm{~min}$.

Such findings are consistent with Wilkinson's (1969b) report that even subjects who are significantly sleep deprived are quite capable of short periods (10-15 min) of mental concentration that far exceed their capacity for normal work shifts (which may last 8 h or more). Thus, a reliance on very short laboratory tests to predict performance decrements in an actual work setting can be dangerous, especially in contexts in which the tolerance for error is quite small (such as in aviation). It may seem possible to compensate for brief test periods by implementing more complex tasks - a possibility that was partially examined here. However, it is evident that such a strategy can backfire and result in decreased test sensitivity, because complex tasks tend to be more interesting than simpler tests and, therefore, less susceptible to fatigue-induced decrements (Elsmore et al,, 1995; Wilkinson, 1969a). This is particularly true in SD paradigms in which sleepy subjects briefly can be returned to "normal" if sufficiently aroused. However, as Wilkinson (1969a) has stated, "the subject's problem is that he habituates to arousing stimuli [quickly] and so this normality cannot be maintained for very long, and hence [the] time factor beats him" (p. 34).

The findings from the present study reaffirm the importance of using increased task duration to heighten test sensitivity, especially when attempting to gain an understanding of the effects of a stressor such as SD. It is concluded that, even when fairly demanding face-valid simulation tasks are employed, at least $30 \mathrm{~min}$ of continuous performance will be required before generalizations to "real-world" work performance can even begin to be made.

\section{REFERENCES}

Caldwell, J. A., Caldwell, J. L., Crowley, J. S., Darling, S. R., WaLlace, S., WoOdrum, L. C., \& JoNes, H. D. (1995). Sustaining female UH-60 helicopter pilot performance with Dexedrine in sustained operations: A simulator study (Tech. Rep. No. 95-27). Fon Rucker, AL: U.S. Army Aeromedical Research Laboratory.

Caldwell, J. A., Caldwell, J. L., Crowley, J. S., Jones, H. D., Darling, S. R., Wallace, S. L., Woodrum. L. C., \& Colon, J. A. (1994). Effects of dextroamphetamine on helicopter pilot performance: A UH-60 simulator study (Tech. Rep. No. 94-43). Fort Rucker, AL: U.S. Army Aeromedical Research Laboratory.

Caldwell, J. A., Jones, R. W., Caldwell, J. L., Colon, J. A., Pegues, A., Iverson, L., Roberts, K., Ramspott, S., Sprenger, W. D., \& GARDNER, S. (1997). The efficacy of hypnotic-induced prophylactic naps for the maintenance of alertness and performance in sustained operations (USAARL Tech. Rep. No. 97-10). Fort Rucker, AL: U.S. Army Aeromedical Research Laboratory. 
Chmiel, N., TotTerdell, P., \& Folkard, S. (1995). On adaptive control, sleep loss, and fatigue. Applied Cognitive Psychology, 9, S39-S53

COMSTOCK, J. R., \& ARNEGARD, R. J. (1992). The multi-attribute task battery for human operator workload and strategic behavior research (NASA Tech. Mem. No. 104174). Hampton, VA: National Aeronautics and Space Administration, Langley Research Center.

DiNGES, D. F. (1989). Napping patterns and effects in human adults. In D. F. Dinges \& R. J. Broughton (Eds.), Sleep and alertness: Chronobiological, behavioral, and medical aspects of napping (pp. 171204). New York: Raven Press.

Elsmore, T. F., HegGe, F. W., Naitoh, P., Kelly, T., Schlangen, K., \& GOMEZ, S. (1995). A comparison of the effects of sleep deprivation on synthetic work performance and a conventional performance as sessment battery (NHRC Rep. No. 95-6). San Diego: Naval Health Research Center.

KRUEGER, G. P. (1989). Sustained work, fatigue, sleep loss, and performance: A review of the issues. Work \& Stress, 3, 129-141.

Pilcher, J. J., \& HuffCutT, A. I. (1996). Effects of sleep deprivation on performance: A meta-analysis. Sleep, 19, 318-326.

WILKINSON, R. T. (196l). Interaction of lack of sleep with knowledge of results, repeated testing, and individual differences. Journal of $E x$ perimental Psychology, 62, 263-271.

WILKINSON, R. T. (1964). Effects of up to 60 hours' sleep deprivation on different types of work. Ergonomics, 17, 175-186.

WiLkinson, R. T. (1969a). Sleep deprivation: Performance tests for partial and selective sleep deprivation. In L. Edwin \& B. F. Riess (Eds.), Progress in clinical psychology: Vol. 3. Dreams and dreaming (p. 2833). New York: Grune \& Stratton.

WILKINSON, R. T. (1969b). Some factors influencing the effect of environmental stressors upon performance. Psychological Bulletin, 72, 260-272.

Wilkinson, R. T., Edwards, R. S., \& Haines, E. (1966). Performance following a night of reduced sleep. Psychonomic Science, 5, 471-472.

Wilkinson, R. T., \& Stretton, M. (1971). Performance after awakening at different times of night. Psychonomic Society, 23, 283-285.

(Manuscript received March 14, 1997 ; revision accepted for publication November 10, 1997.) 